\title{
Dispersion Management for Two-Octave-Spanning High- Energy Sub-Optical-Cycle Parametric Waveform Synthesizers
}

\author{
Shih-Hsuan Chia ${ }^{1-3}$, Giovanni Cirmi ${ }^{1,3}$, Shaobo Fang ${ }^{1,3}$, Giulio M. Rossi ${ }^{1,3}$, Oliver D. Mücke ${ }^{1,3}$, Franz X. Kärtner ${ }^{1-4}$ \\ ${ }^{1}$ Center for Free-Electron Laser Science, Deutsches Elektronen-Synchrotron DESY, Notkestraße 85, 22607 Hamburg, Germany, \\ ${ }^{2}$ Physics Department, University of Hamburg, Luruper Chaussee 149, 22761 Hamburg, Germany \\ ${ }^{3}$ The Hamburg Center for Ultrafast Imaging, Luruper Chaussee 149, 22761 Hamburg, Germany \\ ${ }^{4}$ Department of Electrical Engineering and Computer Science and Research Laboratory of Electronics, \\ Massachusetts Institute of Technology, Cambridge, Massachusetts 02139, USA \\ shih-hsuan.chia@.cfel.de
}

\begin{abstract}
We discuss the dispersion management for multi-mJ 2-octave-wide $(0.5-2.3 \mu \mathrm{m}) 3$ channel parametric waveform synthesizers to support 1.9-fs sub-cycle light transients. 2-octavespanning laser optics are demonstrated, providing an enabling technology for pulse-energy and bandwidth scaling.

OCIS codes: (310.4165) Multilayer design; (190.4970) Parametric oscillators and amplifiers.
\end{abstract}

\section{Introduction}

The emerging field of Waveform Nonlinear Optics, where nonlinear interactions of matter with extremely short optical waveforms custom-tailored within a single cycle of light are studied and controlled, critically depends on the availability of multi-octave-spanning CEP-phase-controlled optical pulses. This is of primary importance, e.g., in the generation of intense isolated attosecond XUV pulses [1], in launching valence-electron wavepacket dynamics in atoms and molecules [2] and the control of sub-cycle electron transport in solids [3].

Recent developments in broadband waveform generation have produced coherent optical spectra with $>1$-octave bandwidth by sub-cycle waveform synthesis [4] and supercontinuum generation [2,5]. In addition, to realize the energy scalability of ultrabroadband synthesizers for the realization of bright coherent tabletop high-harmonic sources in the water-window and $\mathrm{keV}$ X-ray region [6], multi-channel parametric waveform synthesizers with several amplification stages are required [7], which calls for temporal optimization in each amplification stage to maximize both conversion efficiency and bandwidth, as well as to simultaneously suppress superfluorescence backgrounds: The dispersion management for the generation of intense sub-optical-cycle sources is extremely delicate and challenging, especially requiring ultrabroadband precision laser optics. Active compression systems based on spatial light modulators with $>1$-octave bandwidths have been demonstrated [5]. However, they are hampered by the bandwidth and diffraction efficiency of the gratings used in 4- $f$ systems. Therefore, they have not directly been applied to multi-octave-spanning high-intensity sources. Multilayer dielectric coatings such as the ones used for chirped mirrors pairs [8], although not adaptable, have the advantages of supporting potentially multioctave bandwidth with high reflectivity. Therefore, multilayer mirror designs are widely employed as robust solutions: 1.5-octave-wide chirped mirror pairs for pulse compression have been developed, and optical synthesizers with several compressed channels have been realized with separate chirped mirror designs [2]: the pulses covering different spectral ranges are individually compressed before the pulse recombination. However, in that case the pulse energy is limited by the peak power of the combined intense ultrashort pulse, which induces detrimental nonlinearities (i.e., $B$-integral) in the following optical beam path and particularly in the beam combiner optics and vacuum-chamber window. In addition, the dispersion of the beam combiner around the edge of the high reflection band is difficult to be controlled. As a result, synthesized electric-field transients without spectral gaps are hard to be achieved with dichroic mirrors: spectral gaps cannot be avoided in between the channels [2]. Therefore, to efficiently synthesize multiple broadband optical waveforms from different channels, the precise dispersion matching between the transmission and reflection port of the beam combiner are required: broadband dichroic mirrors (DM) with matched dispersion control are necessary but not available so far. In this work, novel mirror designs for 2-octave-spanning waveform synthesizers delivering multi-mJ pulse energy is introduced: We design, fabricate, and characterize the required laser optics, chirped dichroic mirrors (CDMs) for efficient splitting and coherent combining of pulses and ultra-broadband double chirped mirror (DCM) pairs for final compression: to avoid $B$-integral problems, we recombine the still slightly chirped pulses first on the CDMs and then use an ultrabroadband DCM pair as a final compressor unit. Fig. 1 shows the use of these optics in an actual 2-octave-wide 3-channel parametric synthesizer [7]. 


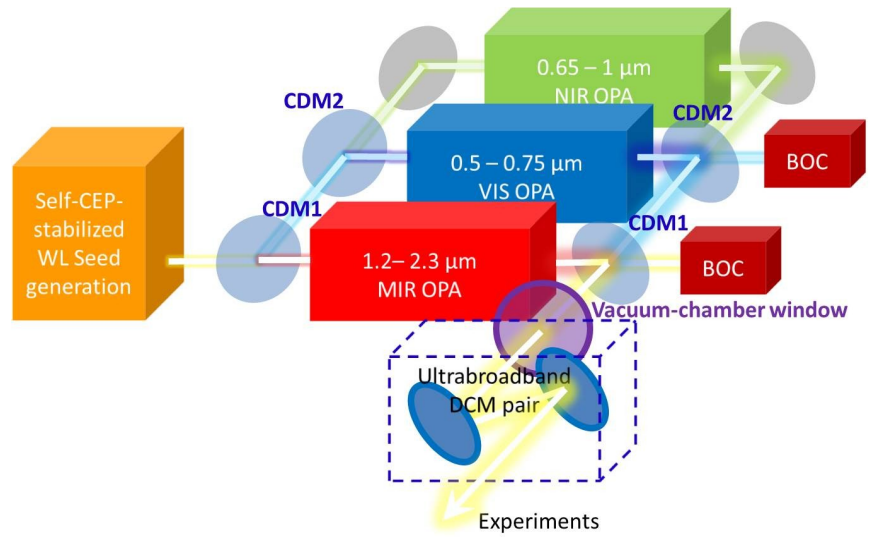

Fig. 1. Scheme of a 2-octave-wide 3-channel parametric synthesizer [6]. CEP, carrier-envelope phase; WL, white-light; CDM, chirped dichroic mirror; OPA, optical parametric amplifier; DCM, double-chirped mirror; BOC, balanced optical cross-correlator.

\section{Dichroic dispersion-controlled scheme}

In principle, chirped mirrors are designed as dispersive optical interference coatings with low- and high-index dielectric layer pairs to achieve dispersion management. However, pronounced group delay (GD) ripples are usually observed especially in broadband designs. The double-chirped structure [8] was proposed to achieve impedance matching by adiabatically tapering the impedance in the front layer pairs, which is shown to be equivalent to an adiabatic chirp in the thickness of the high-index layer in addition to the chirp of the center wavelength of the Bragg mirror. However, multilayer mirror designs covering 2 octaves in bandwidth, which requires 2-octave impedance matching, have not been achieved so far. Here, we introduce a dual-adiabatic-matching (DAM) structure that generates also a double-chirp in the back section of the mirror approaching the substrate, adiabatically tapering the impedance again to provide high transmittance for longer wavelengths. A comparison between the performances of different coating structures are plotted in Fig. 2.

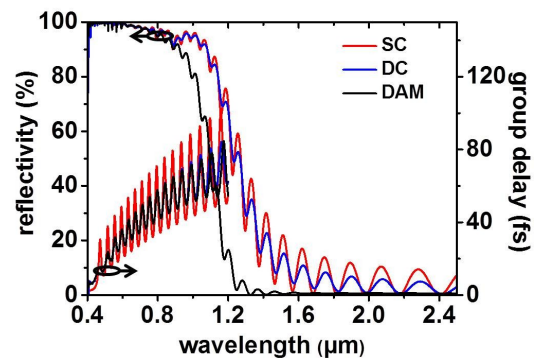

Fig. 2. The reflectivity/GD of different chirped mirror structures. A design of the simple-chirped (SC) structure monotonically increases the Bragg wavelength with quarter-wave layer pairs to provide negative dispersion; the double-chirped (DC) coating tapering the impedance in the front section based on the SC structure features reduced GD ripples in the high-reflectivity range; the proposed DAM structure further introduces another impedance-matching section in the back layers, resulting in high transmittance for longer wavelengths.

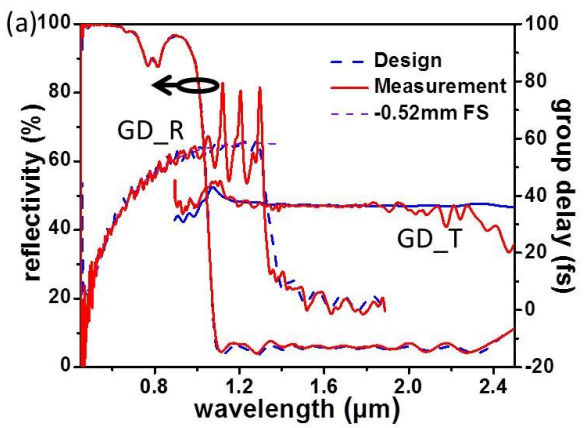

(b)

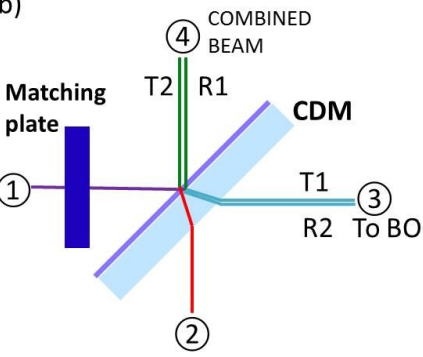

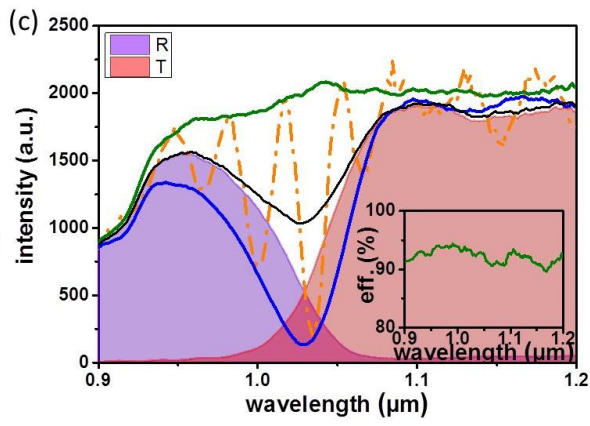

Fig. 3. (a) the designed/measured reflectivity/GD of the chirped dichroic mirror (CDM), as well as the corresponding GD design goals. (b) The schematic diagram of a dispersion-matched system based on the CDM with two input ports, port 1 and port 2. (c) The reflection, transmission, and the combined spectra (green, blue, and orange dashed) in port 4 with different delays between port 1 and port 2 in (b), as well as the incoherently combined spectra (black). (Inset) The optimized beam combining efficiency with the green spectrum is $>90 \%$, even including the $\sim 8 \%$ total interface reflection losses of the matching plate in port 1. GD_R, designed/measured GD in reflection; GD_T, the transmitted designed/measured GD; FS, fused silica.

With the ultrabroadband impedance-matching features shown in Fig. 2, we implement the proposed DAM structure to design a chirped dichroic mirror (CDM). A fast algorithm [9] is employed to meet the design goals for our parametric synthesizer with an initial DAM design. We design the GD in reflection to compensate the dispersion of a $0.52-\mathrm{mm}$-thick fused silica plate in the spectral range of $0.45 \mu \mathrm{m}-1.3 \mu \mathrm{m}$, which is even broader than the high reflectivity range of $0.45 \mu \mathrm{m}-1.1 \mu \mathrm{m}$, as shown in Fig. 3. The GD control over the edge of the reflection band is 
beneficial for the synthesizer application, allowing beam combining with a spectral overlap between different channels: Based on the CDM, a dispersion matched scheme is proposed by inserting a matching fused silica plate in port 1 as shown in Fig. 3(b). The purple/red curves in Fig. 3(c) show the reflected/transmitted spectra measured using a white-light source from port 1 and port 2, respectively. By fine-tuning the optical delay, constructively/destructively interfered spectra over the spectrally overlapping region can be obtained as the green/blue curves, respectively. The beam combining efficiency with the green spectrum is $>90 \%$ over the transition range, even including the $\sim 8 \%$ total interface reflection losses of the silica plate in port 1 . The interfered fringe(s) in the spectral overlapping region is extremely sensitive to the delay between the pulses from the two input ports, which benefits the precise stabilization of the relative timing.

\section{2-octave-wide high reflection double-chirped mirror pairs}

The DAM structure lends itself to the design of an ultrabroadband DCM pair as the final compression unit. The proposed structure particularly reflects the light within its Bragg wavelength and provides smooth transmittance for the longer wavelength, which is a proper impedance matching section to reduce the Gires-Tournois effect in the chirped mirror design. A 2-octave-wide DCM pair is designed, fabricated and characterized to compensate 1.46$\mathrm{mm}$-thick fused silica plates in the spectral range from $0.49 \mu \mathrm{m}-1.05 \mu \mathrm{m}$ and $0.32-\mathrm{mm}$ of $\mathrm{ZnSe}$ in the range of 1.05 $\mu \mathrm{m}-2.3 \mu \mathrm{m}$. As a result, the average reflectivity of the ultrabroadband DCM pair is $>90 \%$ and the calculated peakto-peak values of the residual average GD ripples are less than $5 \mathrm{fs}$ over $>2$-octave bandwidth.
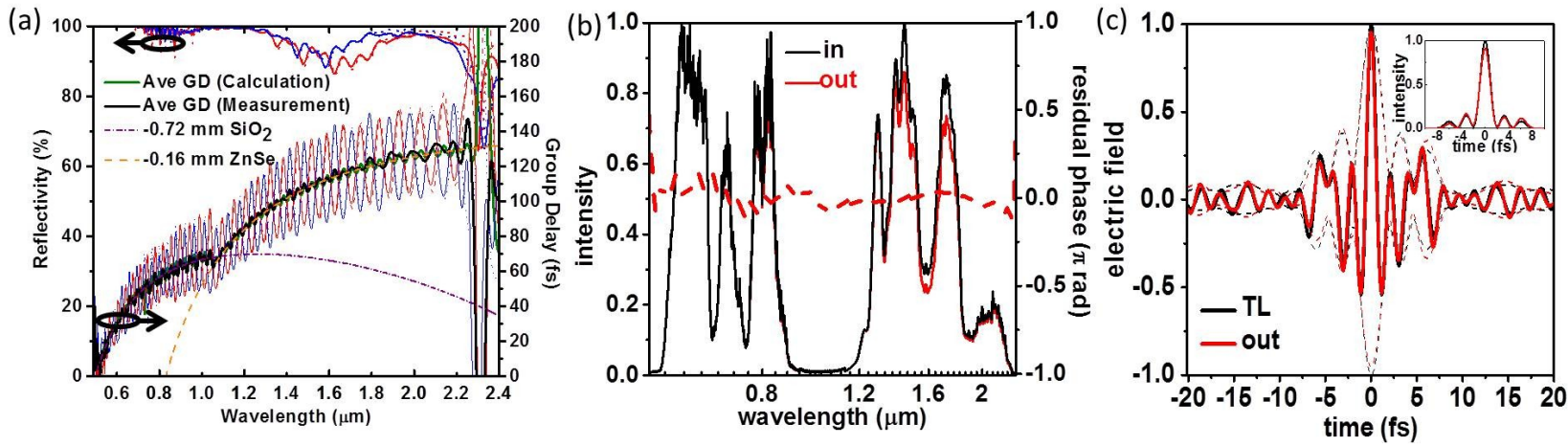

Fig. 4. (a) The reflectivity and GD of the ultrabroadband DCM pair: the calculation is shown as blue/red dashed lines and the solid lines show the measurement results. The pulse characteristics in (b) frequency domain and (c) time domain after the ultrabroadband DCM pair. The black curve in (b) is the measured combined OPA spectra, as well as the transform-limited electric waveform in (c). The red curves show the pulse after the mirror pair, as well as the residual phase shown as dashed line in (b). The duration of the pulses depicted in the inset as black and red lines are $1.88 \mathrm{fs}$ and $1.93 \mathrm{fs}$, respectively. TL, transform-limited electric waveform.

We analyzed the expected pulse distortions introduced by the chirped mirror synthesizer optics using the experimental second-stage OPA output spectra [7] shown in Fig. 4(b). The spectrum below $1 \mu \mathrm{m}$ is reflected by the $\mathrm{CDM}$, and the spectrum above $1.1 \mu \mathrm{m}$ is transmitted. The combined pulse, with $1.88 \mathrm{fs}$ FWHM transform-limited pulse duration, is chirped to decrease the peak intensity when passing through the combiner substrate and the vacuum-chamber window, and the ultrabroadband DCM pair can compensate the dispersion of the combined pulse afterwards. The pulse distortions were examined by considering the measured reflectivity and residual GD errors of the mirrors, as shown in Fig. 4. Fig. 4(b-c) shows the synthesized pulse after compression by the ultrabroadband DCM pair. The total measured residual phase error of the DCM pair, is $<0.1 \pi \mathrm{rad}$ (i.e., $<\lambda / 20$ ), as the red dashed curve in Fig. 4(b) shows, enabling pulse compression very close to its transform limit of 1.93 fs. 2-octave-bandwidth pulse compression can thus be achieved for the first time.

The detailed features of the DAM structure, dispersion-matched scheme, and the ultrabroadband DCM pair will be presented, as well as the individual dispersion management in each amplification stage based on the customdesigned chirped mirrors to achieve temporal optimization between the pump and seed pulses.

\section{References}

[1] E. J. Takahashi et al., Nat. Commun. 4,2691 (2013); E. J. Takahashi et al., Phys. Rev. Lett. 104, 233901 (2010).

[2] A. Wirth et al., Science 334, 195 (2011). Also see: M. T. Hassan et al., Rev. Sci. Instrum. 83, 111301 (2012); T. T. Luu et al., QELS 2013, paper QF1C.6 (2013).

[3] O. D. Mücke, Phys. Rev. B 84, 081202(R) (2011).

[4] S.-W. Huang et al., Nat. Photon. 5, 475 (2011).

[5] E. Matsubara et al., JOSA B 24, 985 (2007).

[6] T. Popmintchev et al., Science 336, 1287 (2012).

[7] G. M. Rossi et al., in CLEO: 2014, OSA Technical Digest (online) (Optical Society of America, 2014 ), paper SF1E.3.

[8] F. X. Kärtner Opt. Lett. 22, 831 (1997); F. X. Kärtner et al., J. Opt. Soc. Am. B 18, 882 (2001).

[9] J. R. Birge et al., Appl. Opt. 46, 2656 (2007). 